\title{
Exact Analysis of the Main Bistatic Scattering Area on Sea Surface
}

\author{
Xianmao $\mathrm{Li}^{1,2}$ \\ 1.The department of \\ Electronics and Information \\ Engineering, Huazhong \\ University of Science and \\ Technology \\ 2.College of Electronics \\ Engineering \\ Naval University of Engineering \\ Wuhan, China \\ e-mail: xianmao916@163.com
}

\author{
Gaoming Huang \\ College of Electronics Engineering \\ Naval University of Engineering \\ Wuhan, China \\ e-mail: redforce@sohu.com
}

\author{
Tianlin Dong \\ The department of \\ Electronics and \\ Information \\ Engineering, Huazhong \\ University of Science and \\ Technology \\ Wuhan, China \\ e-mail: dtlHust@hust.edu
}

\begin{abstract}
The acertainment and calculation of the bistatic main scattering area on ocean is the basis for the calculation of the energy entering the receiver when scattered on the sea wave scattering. The paper main studies analysis and calculation of scattering area when transmitter and receiver are onboard or airborne. Mainly analysis and calculation of distribution and acreage of the main scattering area while transmitter and receiver are all onboard, transmitter is onboard and receiver is airborne, or transmitter and receiver are all airborne. Studies the calculation of all kind typical main scattering areas when radar transmitt microwave and it is received by reconnaissance by simulation.
\end{abstract}

Keywords-component; sea scattering, main scattering area, bistatic, irradiated region, radar, reconnaissance

\section{INTRODUCTION}

In the calculation of sea electromagnetic wave, we should consider the transmitted beam and received beam, both of them should shot sea, and they should have overlap region in the irradiated region on sea, then the scattering electromagnetic waves of the transmitter can be detected by the receiver. Define scattering intensity reference value 0dBreFS[1], it's refers to scattering signal strength on sea surface in certain direction which ensures the scattering enagy can be received by the receiver's antenna. Definition of main scattering area: Refer to receiver, transmitter radiating electromagnetic wave, it is scattered by sea surface, when signal strength per unit acreage that is can be received by receiver antenna is exceeded a certain value, all the points satisfying condition compose an area, it is namely main scattering area. In the certain condition here, we define the main area that while transmitter radiates the sea surface, the point's scattering dentity to reciever is the most trength, the scattering strength per unit area on sea defines 0dBreFS, when the scattering in anyone point exceeds $-10 \mathrm{~dB}$ (relative to 0dBreFS), all the points compose main scattering area.

Assuming the transmitter antenna is directional beam, and the receiver antenna is directional beam too, the gain gradually reduces in the beam edge, when the edge gain reduce to the tenth of max gain in the center is the edge of main irradiation area, the intersection area of transmitter antenna's main irradiation and the receiver antenna's main irradiation on the sea is the approximate main scattering area in the engineering sense. The paper studies analysis and calculation of the main scattering area for various transmitters and receivers on the sea or in the air, including sea-based transmitter and receiver, sea-based transmitter and air-based receiver, air-based transmitter and receiver. It becomes the basis for the calculation of energy received by the receiver in case of sea scattering.

\section{ANALYSIS THE DISTRIBUTION OF MAIN SCATTERING AREA}

According to power of sea point scattering to receiver determine and shape and size of the main sea scattering area.

The main scattering area's size and shape is related to the sea conditions, and the signals of the sea distribute within a certain range, not completely uniformed. The beam of receiver is also directional, the beam when point to the sea forms a region on the sea, shown in the Fig.1, Fig.2, and the distribution is not entirely average on the area, shown in the Fig.2.

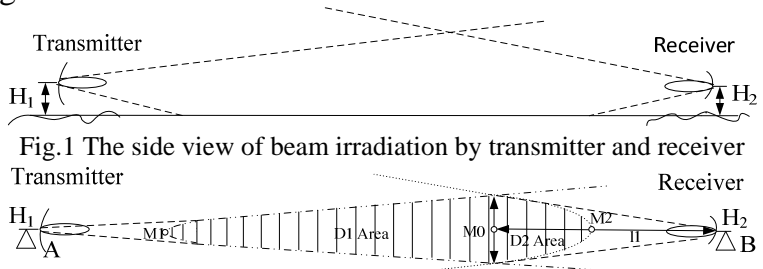

Fig.2 The intersection area of transmiting beam and receiving beam

The erect lines filling area is the area A that the scattering electronicmegnatic wave can be received by receiver. Considering the sea scattering, the area $\mathrm{A}$ is main scattering area.

The influence of the receiver of sea scattering depends on, the beam of transmitter exposure on the area and the receiver's receiving area,only in the over lapping area, the sea scattering wave is effective to the receiver, the effective area is shown as area D1 and D2 in Fig.2. To take the typical sea search radar antenna direction graph as an example, the transmitter antenna of radar and the receiver antenna are all 
use the unidirectional antenna model because of transmiter and receiver are independent.

The example using the sea search radar antenna direction graph, the horizontal is gauss beam, the pitching is radar antenna of $\operatorname{CSC}^{2}$ antenna, hypothesis the azimuth is $\theta$, the pitching angle is $\varphi$,mainly consider the antenna's main lobe pattern.

(1)Horizontal antenna direction graph model is:

$g(\theta)=\exp \left(-\zeta \theta^{2}\right)$

In the formula $\zeta=\frac{4 \ln \sqrt{2}}{\theta_{0.5}^{2}}$, and

$\theta_{0.5}$ the beam width of half power in one way

(2)The pitching antenna direction graph model is:

$f(\varphi)=\frac{\sin \left(0.5 \varphi_{0.5}+\varphi_{0}\right)}{\sqrt{2} \sin \varphi_{e}}$

$\varphi_{e}$ - the pitching angle of radar

$\varphi_{0.5}$ the half power width of pitching direction graph

$\varphi_{c}$ — the max pitching angle of the direction graph, namely the angle of main lobe direction relate to the horizontal plane.

So, the gain of $\mathrm{CSC}^{2}$ bungalow antenna is the antenna pattern function multiplying the max gain[2]:

$$
\begin{aligned}
& G(\theta, \varphi)=\eta G_{\max } g(\theta) f(\varphi) \\
& =\eta G_{\max } \exp \left[-\zeta\left(\theta-\theta_{0}\right)\right] \frac{\sin \left(0.5 \varphi_{0.5}+\varphi_{c}\right)}{\sqrt{2} \sin (\varphi)}
\end{aligned}
$$

The receiver's antenna using the Sinc Figure pattern function[3]。

$$
F(\theta, \varphi)=F_{\max } \frac{\sin \left(2.78 \frac{\theta-\theta_{0}}{\theta_{0.5}}\right) \sin \left(2.78 \frac{\varphi-\varphi_{0}}{\varphi_{0.5}}\right)}{\left(2.78 \frac{\theta-\theta_{0}}{\theta_{0.5}}\right)\left(2.78 \frac{\varphi-\varphi_{0}}{\varphi_{0.5}}\right)}
$$

Form the general sense, the main scattering area is the lapping area of radar exposure area and the receiver's accept area.

\section{A. The condition that transmitter and receiver are all onboard}

Form the Fig. 3 we know the main scattering area must lay between the transmitter and receiver. Assuming point A is origin, the $\mathrm{X}$-axis is the line lining the transmitter and the receiver, set a line through the transmitter and perpendicular to X-axis as Y-axis. First, we should confirm the transmitter antenna's initial point $M_{1}$ on X-axis and the receiver antenna's initial point $M_{2}$. Assume the transmitter antenna's pitch axial is zero, the point that the antenna beam low fringe reachs the sea is $M_{1}$, as the same, assume the receiver antenna's pitch axial is zero, the point that the antenna beam low fringe reachs the sea is $M_{2}$. The lapping area is the main scattering area between $M_{1}$ and $M_{2}$. Divides it to two cases. Case 1: the antenna of transmiter and antenna of receiver is directly faced, shown as Fig.3; case 2: the antenna of transmiter and antenna of receiver is not directly faced, but they have lapping area. Assume $L=|A-B|$.

First, we analysis the case 1 . Because the intersection point $M_{0}$ is for away from point $\mathrm{A}$ and point $\mathrm{B}$, we can approximate think in point $M_{0}$, the horizontal beam width is $\theta_{0.5}$. Assume the distance between $M_{1}$ and $M_{2}$ is $d$, assume

$$
\begin{aligned}
l_{1}= & \left|M_{1}-A\right|, \text { so } l_{1}=\frac{H_{1}}{\operatorname{tg} \frac{\varphi_{0.5}}{2}} \cdot \\
d & =2 r \cdot \operatorname{tg} \frac{\varphi_{0.5}}{2}=2 \sqrt{H_{1}^{2}+l_{1}^{2}} \cdot \operatorname{tg} \frac{\varphi_{0.5}}{2} \\
r & =\sqrt{H_{1}^{2}+l_{1}^{2}} \\
d & =2 r \cdot \operatorname{tg} \frac{\theta_{0.5}}{2}=2 \sqrt{H_{1}^{2}+l_{0}^{2}} \cdot \operatorname{tg} \frac{\theta_{0.5}}{2}
\end{aligned}
$$

The point of intersection of surface exposure width:

$d_{0}=2 \sqrt{H_{1}^{2}+l_{0}^{2}} \cdot \operatorname{tg} \frac{\theta_{1,0.5}}{2}=2 \sqrt{H_{2}^{2}+\left(L-l_{0}\right)^{2}} \cdot \operatorname{tg} \frac{\theta_{2,0.5}}{2} \mathrm{ma}$ ke $(k-1) l_{0}^{2}+2 L l_{0}+\left(k H_{1}^{2}-H_{2}^{2}-L^{2}\right)=0$, and

$$
k=\left[\frac{\operatorname{tg}\left(\theta_{1,0.5} / 2\right)}{\operatorname{tg}\left(\theta_{2,0.5} / 2\right)}\right]^{2}
$$

Can get:

$$
l_{0}=\frac{-2 L \pm \sqrt{4 L^{2}-4(k-1)\left(k H_{1}^{2}-H_{2}^{2}-L^{2}\right)}}{2(k-1)}
$$

Supposing the angle between two ends of line $d$ to point $\mathrm{B}$ is $\theta_{1}$, the distance is $r \circ r$ and horizontal plane angle is $\alpha_{1}$, for get $d, \theta_{1}$ should be calculate.

1) aim at the transmitter antenna,when $\theta_{0}=0, \alpha_{0}=0$,get the edge of beam when the gain of main lobe down to the $1 / 10$ of max gain, make $G\left(\theta_{1}, \varphi_{1}\right)=\frac{1}{10} G_{\max }$ ,can get:

$$
\theta_{1}=\left[\frac{1}{\zeta} \ln \operatorname{Sin}\left(0.5 \varphi_{0.5}+\varphi_{c}\right)-\frac{1}{\zeta} \ln \left(\frac{\sqrt{2} \sin \varphi_{1}}{10 \eta}\right)\right]^{1 / 2}
$$

2) aim at receiver antenna, when $\theta_{0}=0, \alpha_{0}=0$, get the edge of beam when the gain of main lobe down to the 1/10 of max gain, then make $F\left(\theta_{1}, \varphi_{1}\right)=\frac{1}{10} F_{\max }$,get the formula base above condition:

$$
\frac{\sin \left(2.78 \frac{\theta_{1}}{\theta_{0.5}}\right)}{\left(2.78 \frac{\theta_{1}}{\theta_{0.5}}\right)}=\frac{0.278 \pi \varphi_{1}}{\varphi_{0.5}} \cdot \frac{1}{\sin \left(2.78 \frac{\varphi_{1}}{\varphi_{0.5}}\right)}
$$


So we can get $\theta_{1}$ from formula(3) by computer calculation.

$$
d=2 r \cdot \operatorname{tg} \theta_{1}
$$

Condition first, when the devices is directly faced, the overlaping area is the main scattering area, the shape is like the shadow part shown in the Fig.2.

The acreage of area $D_{1}$ is from $M_{1}$ to $M_{0}, d$ changes follow $x$,indirectly $d$ is function by $x$. Using intergration gets the area acreage from $M_{1}$ to $M_{0}, D_{1}=\int_{M_{1}}^{M_{0}} d(x) d x$. As the same, using intergration to get the area acreage from $M_{0}$ to $M_{2} \quad D_{2}=\int_{M_{2}}^{M_{0}} d(x) d x$. So, the area acreage of main scattering is $A_{0}=\int_{M_{1}}^{M_{0}} d(x) d x+\int_{M_{0}}^{M_{2}} d(x) d x$.

Condition second, when the devices is not directly faced, the overlaping area is the main scattering area, the shape is like the shadow part shown in the Fig. 3.

A

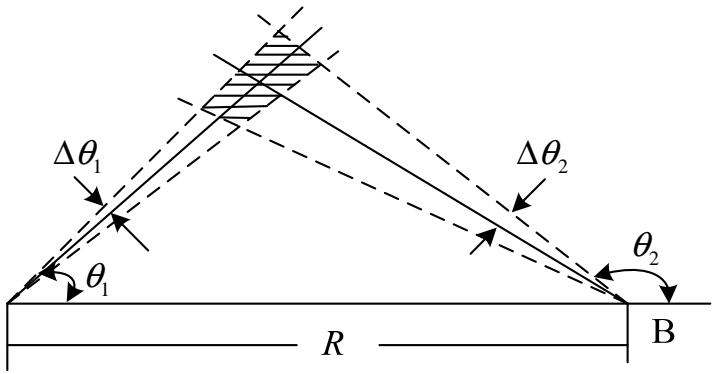

Fig.3 The effective scattering area when devices are not directly faced

We can calculate the acreage of the area by following formula(4)[4].

$$
A_{0}=\left|\frac{\Delta d_{1} \Delta d_{2}}{\sin \left(\theta_{2}-\theta_{1}\right)}\right|=\left|\frac{4 R^{2} \Delta \theta_{1} \Delta \theta_{2}}{\sin \theta_{1} \sin \theta_{2} \sin \left(\theta_{2}-\theta_{1}\right)}\right|
$$

\section{B. Condition that transmitter id onboard and receiver} is airborne

when the transmitter is onboard and receiver is airborne,, the overlaping area is the main scattering area approximately, and it will show two conditions.

Condition first, the exposure area of receiver antenna is included in the transmitter exposure area to the sea, the shape is like the shadow part shown in the Fig.4.

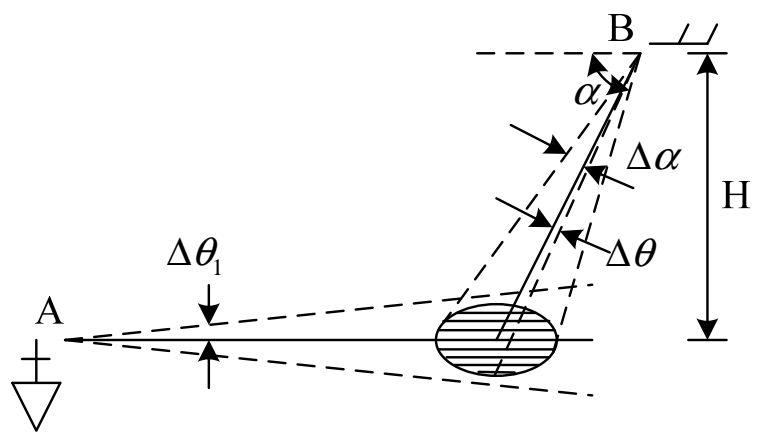

Fig.4 The exposure area of receiver antenna is included in the transmitter exposure area to the sea[4]

$$
A_{0}=4 R \Delta \alpha \cdot R \Delta \theta=\frac{4 H^{2}}{\sin ^{2} \alpha} \Delta \alpha \cdot \Delta \theta
$$

Condition second, the exposure area of receiver antenna and the transmitter exposure area have overlap region, considering the condition that overlap has the max area the shape like the shadow part shown in the Fig.5. The beam of transmitter antenna has a narrow angle, then the two line of the beam laying in the ellipse can be consider as parallel lines.

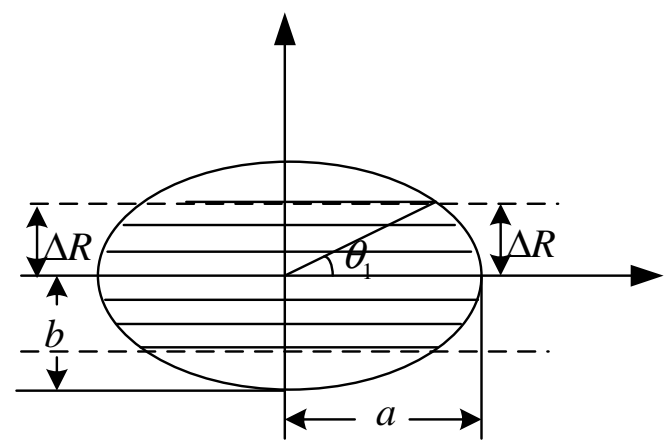

Fig. 5 The exposure area of receiver antenna and the transmitter exposure area have overlap region

$$
R=\frac{H}{\sin \alpha}, a=\frac{2 R \Delta \alpha}{\sin \alpha}, b=2 R \Delta \theta, \Delta R=R_{1} \sin \left(\Delta \theta_{1}\right),
$$
$\theta_{1}=\arcsin (\Delta R / b)$

$R_{1}$ is the distance between transmiter and the center of the ellipse, $\Delta R$ is the distance of approximate parallel area boundary lines of transmitter beam on sea surface to X-axis. We can calculate the acreage of the area by following formula(5)[5].

$$
A_{0}=\pi a b-2 a b \arccos \frac{y}{b}-x y
$$

Thereinto, $x=\Delta R / \operatorname{tg} \theta_{1}, y=\Delta R$.

\section{Condition that transmitter and receiver are all airborne}

when the transmitter is airborne and the receiver is airborne, the overlaping area is the main scattering area, the largest overlaping area is commonly shown like the shadow part in the Fig.6. The ellipse center coincidence, but the long axis's direction is different. The special case is inclusive relation, the small ellipse's area is the overlaping area $A_{0}=\pi a b$, when the ellipses are not inclusive relation, shown as Fig.6. 


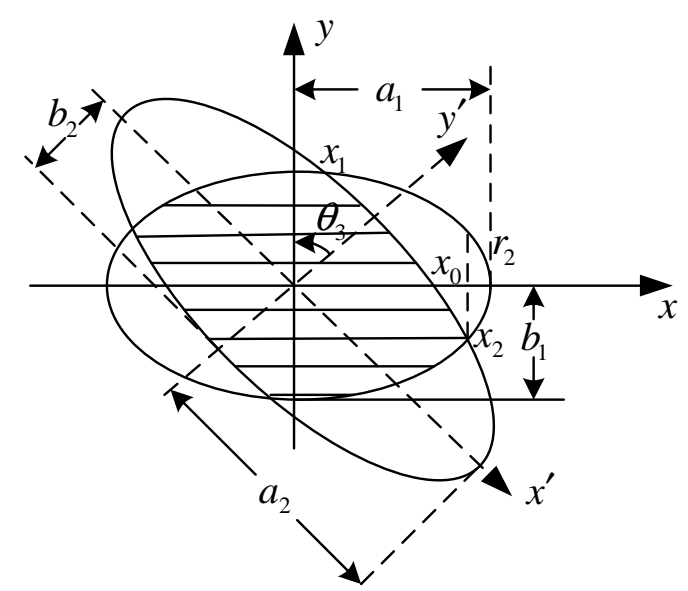

Fig.6 The main scattering area of air transmitter to air receiver

Suppose $(x, y)$ is the intersect points of the two ellipses, through the transformation of two coordinates, $(x, y)$ is transformed to $\left(x^{\prime}, y^{\prime}\right)$ in another coordinate, and we can get formulae as follows:

$$
\begin{gathered}
x^{\prime}=\left(x^{2}+y^{2}\right) \cos ^{2}\left[\theta_{3}+\operatorname{arctg}(y / x)\right] \\
y^{\prime}=\left(x^{2}+y^{2}\right) \sin ^{2}\left[\theta_{3}+\operatorname{arctg}(y / x)\right] \\
\frac{x^{2}}{a_{1}^{2}}+\frac{y^{2}}{b_{1}^{2}}=1 \\
\frac{x^{\prime 2}}{a_{2}^{2}}+\frac{y^{\prime 2}}{b_{2}^{2}}=1
\end{gathered}
$$

Though simultaneous equations (6)-(8), we can get:

$$
\begin{aligned}
& \frac{\left[x^{2}+b_{1}^{2}-\frac{b_{1}^{2} x^{2}}{a_{1}^{2}}\right]}{a_{2}^{2}} \cdot \cos ^{2}\left(\theta_{3}+\operatorname{arctg}\left[\frac{ \pm b_{1} \sqrt{1-x^{2} / a^{2}}}{x}\right]\right)+ \\
& \frac{\left[x^{2}+b_{1}^{2}-\frac{b_{1}^{2} x^{2}}{a_{1}^{2}}\right]}{b_{2}^{2}} \cdot \sin ^{2}\left(\theta_{3}+\operatorname{arctg}\left[\frac{ \pm b_{1} \sqrt{1-x^{2} / a^{2}}}{x}\right]\right)=1
\end{aligned}
$$

$$
y= \pm b_{1} \sqrt{1-x^{2} / a_{1}^{2}}
$$

Adopting computer approach value to formula (10), we can calculate four value for $\mathrm{x}$, and adopting formula (11), we can calculate four corresponding value for $\mathrm{y}$. and then they can form four groups values of $(x, y)$, and they are the intersecting points.
Adopting integral method to calculate area, can get calculation formula as follows:

$$
\begin{aligned}
& A_{0}=\pi a b-2 \int_{x_{1}}^{x_{2}} b_{1} \sqrt{1-\frac{x^{2}}{a_{1}^{2}}} \pm M(x) d x \\
& -2\left(a_{1} b_{1} \arccos \frac{x_{2}}{a_{1}}-x_{2} b_{1} \sqrt{1-x_{2}^{2} / a_{1}^{2}}\right)
\end{aligned}
$$

thereinto:

$$
\begin{aligned}
& M(x)= \\
& \sqrt{\left[x^{2}+b_{1}^{2}\left(1-\frac{x^{2}}{a_{1}^{2}}\right)\right] \sin \left[\theta_{3}+\operatorname{arctg}\left(\frac{ \pm b_{1} \sqrt{1-x^{2} / a_{1}^{2}}}{x}\right)\right]}
\end{aligned}
$$

In basis of Fig.6 peculiarity, we can calculate intersect point $\left(x_{0}, 0\right)$ between ellipse 2 and $\mathrm{x}$ axis positive direction. Basis of follow formula

$$
\begin{aligned}
& {\left[x^{2}+b_{1}^{2}\left(1-\frac{x^{2}}{a_{1}^{2}}\right)\right] \cdot} \\
& \sin \left[\theta_{3}+\operatorname{arctg}\left(\frac{ \pm b_{1} \sqrt{1-x^{2} / a_{1}^{2}}}{x}\right)\right]=0
\end{aligned}
$$

Can get $x_{0}=\frac{b_{1}}{\sqrt{b_{1}^{2} / a_{1}^{2}+\operatorname{tg}^{2} \theta_{3}}}$, and then we can get area formula as follows by formula(12):

$$
\begin{array}{r}
A_{0}=\pi a b-2 \int_{x_{1}}^{x_{0}} b_{1} \sqrt{1-\frac{x^{2}}{a_{1}^{2}}}-M(x) d x \\
-2 \int_{x_{0}}^{x_{2}} b_{1} \sqrt{1-\frac{x^{2}}{a_{1}^{2}}}+M(x) d x \\
-2\left(a_{1} b_{1} \arccos \frac{x_{2}}{a_{1}}-x_{2} b_{1} \sqrt{1-x_{2}^{2} / a_{1}^{2}}\right)
\end{array}
$$

III. SIMULATION

Composing transmiter is radar and receiver is reconnaissance.

\section{A. The condition of onboard transmitter and onboard receiver}

Assume point $\mathrm{A}$ is radar, point $\mathrm{B}$ is receiver, the height of point $A$ is 30 meter, the height of point $B$ is 30 meter too. The distance between A、B is 20 kilometer. The antenna of radar A is CSC bungalow, the receiver antenna B is Sinc Figure. The main lobe horizontal beam width of antenna A is $5^{\circ}$, the pitching beam width is $30^{\circ}$; The main lobe horizontal beam width of antenna $\mathrm{B}$ is $20^{\circ}$, the pitching beam width is $20^{\circ}$.

Condition first, when the devices is directly faced, the overlaping area is the main scattering area, the shape is like 
the shadow part shown in the Fig.2. The effective scattering area is shown as Fig.7, area is 51.7 square kilometers.

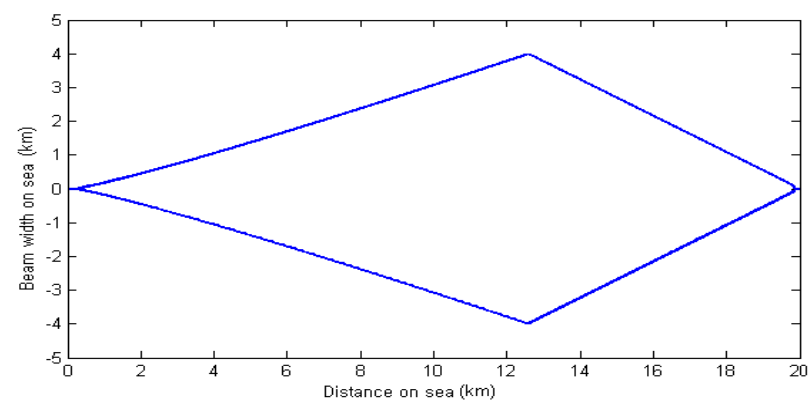

Fig.7 The effective area when transmitter antenna directly face to the receiver antenna

Condition second,when the devices is not directly faced, the overlaping area is the main scattering area, the shape is like the shadow part shown in the Fig.3.

$$
\begin{aligned}
& \text { When } \theta_{1}=45^{\circ}, \theta_{2}=150^{\circ}, \text { according to the } \\
& \text { simulation results in the further area, } \\
& \Delta \theta_{1}=4.16^{\circ}, \Delta \theta_{2}=16.6^{\circ}, A_{0}=98.6 \text { square kilometers. }
\end{aligned}
$$

\section{B. Condition that transmitter is onboard and receiver \\ is airborne}

Condition first, the transmitter is on sea and the receiver is on air, the overlaping area is the main scattering area, the shape is like the shadow part shown in the Fig.4.

Assume $H_{2}=12000 m$ according to the simulation results(1) in the further area, $\alpha=70^{\circ}, \Delta \alpha=\Delta \theta=16.6^{\circ}, \Delta \theta_{1}=4.16^{\circ}, A_{0}=54.7$ square kilometers.

Condition second, the exposure area of receiver antenna and the transmitter exposure area have overlap region, the shape like the shadow part shown in the Fig.5.

Assume $H_{2}=12000 m$ according to the simulation results(1) in the further area, $\alpha=70^{\circ}, \Delta \alpha=\Delta \theta=16.6^{\circ}, R_{1}=13000 \mathrm{~m}, \Delta \theta_{1}=4.16^{\circ}$, $A_{0}=21.8$ square kilometers.

\section{Condition that transmitter and receiver are all airborne}

when transmitter and receiver are all airborne, the overlaping area is the main scattering area, when the ellipses are not inclusive relation, shown as Fig.6.

Assume $H_{1}=12000 \mathrm{~m}$ according to the simulation results(1) in the further area, $\alpha_{1}=70^{\circ}, \Delta \alpha_{1}=\Delta \theta_{1}=16.6^{\circ}$. Assume $H_{2}=7200 m$ according to the simulation results(1) in the further area, $\alpha_{2}=40^{\circ}, \Delta \alpha_{2}=15^{\circ}, \Delta \theta_{2}=4.16^{\circ}, \theta_{3}=50^{\circ}$, so $A_{0}=37.2$ square kilometers.

\section{CONCLIUSION}

The paper analyses different cases of main scattering area, through the geometric analysis and simulation calculation, laid a foundation for the calculation of the energy of receiver on bistatic sea surface scattering. It will play a important role to onboard or airborne reconnaissance, calculation for sea double base radar clutter and single base radar clutter.

\section{REFERENCES}

[1] Smith, J.R., Jr.; Burkholder, R.J. Channeling phenomenon in electromagnetic forward scattering at low grazing, Aug. 2004

[2] Guoyu Wang, Lian-dong Wang,etc.Digital Simulation And Evaluation of Radar EW System[M]. Beijing: National Defence Industry Press, 2004, P85-86

[3] Wanhai Yang. Modeling and Simulation of Radar System[M] . Sian: Xidian University Press, 2007, P157

[4] Guopei Shao,Zhiyao Cao etc.Analysis the Efficiency of ECM Warfare. Beijing: PLA Press, 1998, P59

[5] Math Manual Compile Group. Math Manual. Beijing: Higher EducationPress, 1979, P349-354

[6] Qixiao Ye, Yonghuan Shen. Applied Math Manual (Second Press). Beijing: Science Press, 2006, P65-70 